brain metastases (BMs) were disproportionately excluded from the pioneering trials, herein we evaluated the overall survival (OS) associated with ICI in NSCLC BMs nationally. METHODS: Patients newly-diagnosed with stage 4 NSCLC, including BMs, from 2010-2016 were identified from the National Cancer Database (comprising $>70 \%$ of all newly-diagnosed cancers in the U.S.) Post-approval, median time from diagnosis to ICI was 58days, and this timepoint was selected for all landmark survival analyses (logrank test and multivariable Cox regression) and for multivariable logistic regression to identify predictors of ICI utilization. RESULTS: 50,858 patients presented with advanced NSCLC that involved the brain: representing $27.6 \%$ of all newly-diagnosed stage 4 cases. Following initial FDA approvals in 2015 , ICI rates for BM patients rose from $7.2 \%$ in 2015 to $12.7 \%$ in 2016. OS for NSCLC BMs for patients diagnosed post-approval (median 6.3 months, 95\%CI: 6.0-6.6) was substantially better than those diagnosed pre-approval (median 5.5 months, 95\%CI: 5.4-5.7, p< 0.001 ) and, in fact, than those diagnosed in 2014 (median 5.9 months, $95 \% \mathrm{CI}$ 5.6-6.1, $\mathrm{p}=0.002$ ). Among patients diagnosed post-approval, ICI receipt demonstrated substantially improved OS in landmark survival analyses (median 13.8 months, $95 \%$ CI: $12.2-15.1$; vs. 8.5 months, $95 \%$ CI: $8.3-8.9$, $\mathrm{p}<0.001$ ); benefits which persisted in multivariable landmark survival analyses (HR $0.83,95 \%$ CI: $0.71-0.96, p=0.02$ ), independent of patient characteristics, other therapies, and extracranial disease. For patients diagnosed post-approval, who reached the landmark timepoint, ICI receipt was independent of patient demographics, socioeconomic status, and hospital type - with the exception of Medicaid-insured patients, who were less likely than privately-insured patients to receive ICI (OR $0.77,95 \%$ CI: $0.60-0.97$, $\mathrm{p}=0.03$ ). CONCLUSIONS: Nationally, the dramatic OS benefits of ICIs for advanced NSCLC were also demonstrated for patients with BMs.

\section{IMMU-03. EFFECT OF COMBINING IRRADIATION AND ANTI-CD47 TREATMENT ON THE PHAGOCYTOSIS OF NORMAL} BRAIN CELLS

Forrest Hamrick, Osama Youssef, and Samuel Cheshier; University of Utah, Salt Lake City, UT, USA

Immunotherapy using CD47 blockade has shown promise in treating adult and pediatric malignant primary central nervous system tumors. We recently demonstrated that irradiation significantly enhanced anti-CD47dependent phagocytosis of high-grade glioma cells in vitro. Furthermore, mice engrafted with high-grade human glioma that received anti-CD47 combined with irradiation showed a significant increase in the survival rate and a significant decrease in tumor growth than those that received a single treatment. However, the use of irradiation may result in potential toxicity to normal CNS cells that are not susceptible to macrophage phagocytosis in anti-CD47 monotherapy. We have now extended these studies to test the effect of combining anti-CD47 and irradiation on macrophage-mediated phagocytosis of central nervous system cells. We analyzed phagocytosis of normal human neural stem cells exposed to different irradiation doses in combination with anti-CD47 treatment to assess for the potential toxicity that uniquely exists with this treatment combination.

\section{IMMU-04. IMMUNE-RELATED ADVERSE EVENTS STRONGLY PREDICT SUPERIOR OUTCOMES IN BRAIN METASTASES PATIENTS RECEIVING LOCAL TREATMENT AND IMMUNE CHECKPOINT INHIBITORS \\ Alexander Hulsbergen ${ }^{1}$, Asad Lak ${ }^{1}$, Yu Tung Lo ${ }^{1}$, Nayan Lamba ${ }^{2}$ Steven Nagtegaal ${ }^{3}$, Arun Job ${ }^{1}$, Joost Verhoeff ${ }^{3}$, Timothy Smith ${ }^{4}$, Marike Broekman ${ }^{5}$, and John Phillips ${ }^{1} ;{ }^{1}$ Brigham and Women's Hospital, Boston, MA, USA, ${ }^{2}$ Massachusetts General Hospital, Boston, MA, USA, ${ }^{3}$ University Medical Center Utrecht, Utrecht, Netherlands, ${ }^{4}$ Department of Neurosurgery, Brigham and Women's Hospital, Boston, MA, USA, ${ }^{5}$ Haaglanden Medical Center, The Hague, Netherlands}

INTRODUCTION: In several cancers treated with immune checkpoint inhibitors (ICIs), a remarkable association between the occurrence of immune-related adverse events (irAEs) and superior oncological outcomes has been reported. This effect has hitherto not been reported in the brain. This study aimed to investigate the relation between irAEs and outcomes in brain metastases (BM) patients treated with both local treatment to the brain (LT; i.e. surgery and/or radiation) and ICIs. METHODS: This study is a retrospective cohort analysis of patients treated for non-small cell lung cancer (NSCLC) BMs in a tertiary institution in Boston, MA. Outcomes of interest were overall survival (OS) and intracranial progression-free survival (IC-PFS), measured from the time of LT. Sensitivity analyses were performed to account for immortal time bias (i.e., patients who live longer receive more cycles of ICIs and thus have more opportunity to develop an irAE). RESULTS: A total of 184 patients were included; $62(33.7 \%)$ were treated with neurosurgical resection and $122(66.3 \%)$ with upfront brain radiation. irAEs occurred in 62 patients $(33.7 \%)$. After adjusting for lung-Graded Prognostic Assessment, type of LT, type of ICI, newly diagnosed vs. recurrent $\mathrm{BM}, \mathrm{BM}$ size and number, targetable mutations, and smoking status,
irAEs were strongly associated with better OS (HR $0.33,95 \%$ CI $0.19-$ $0.58, \mathrm{p}<0.0001$ ) and IC-PFS (HR $0.41 ; 95 \%$ CI $0.26-0.65 ; \mathrm{p}=0.0001$ ). Landmark analysis including only patients who received more than 3 cycles of ICI $(n=133)$ demonstrated similar results for OS and IC-PFS, as did sensitivity analysis adjusting for the number of cycles administered (HR range $0.36-0.51$, all p-values < 0.02). CONCLUSIONS: After adjusting for known prognostic factors, irAEs strongly predict superior outcomes after LT in NSCLC BM patients. Sensitivity analysis suggests that this is unlikely due to immortal time bias.

\section{IMMU-05. CSF METABOLOMICS AS A PREDICTOR OF SURVIVAL IN PATIENTS WITH BRAIN METASTASIS RECEIVING IMMUNOTHERAPY \\ Sasmit Sarangi, Devin Zhang, Anita Dong, Edwin Lok, and Eric Wong;}

Beth Israel Deaconess Medical Center, Boston, MA, USA

INTRODUCTION: Checkpoint inhibitors show a positive intracranial response in around $20-50 \%$ of patients with metastatic melanoma and nonsmall cell lung cancer (NSCLC). There is a current paucity of biomarkers to prospectively identify these patients. We hypothesize that CSF would provide useful data on tumor-related metabolites to help stratify responders and non-responders. We focused on adenosine and related nucleotides based on their role in creating an immunosuppressive tumor microenvironment. METHODS: Fifty-one patients ( 43 with melanoma and 8 with NSCLC) received immunotherapies. The CSF samples from this cohort were compared to 30 controls and 60 non-immunotherapy-treated patients (13 with melanoma and 47 with NSCLC) using liquid chromatography/ tandem mass spectrometry for quantitative assessment of metabolites. RESULTS: The age and sex distribution were comparable in both cohorts. The most common immunotherapy regimens were ipilimumab, $\alpha$-interferon and IL-2, while the most common non-immunotherapy regimens were carboplatin/taxol, carboplatin/pemetrexed and pemetrexed alone. CSF AMP, but not adenosine, ADP or ATP, from the immunotherapy cohor was higher in comparison to the non-immunotherapy cohort $(\mathrm{P}=0.0495)$. An adenosine/AMP ratio (AAR) was used to quantify relative adenosine production from AMP. AAR of 3.5-7 was associated with lower median survival from diagnosis of brain metastasis in immunotherapy-treated patients (9.1 months, log-rank $\mathrm{P}=0.0197)$. AAR below or above this range had significantly higher median survival (27.7 and 24.2 months, respectively). The same AARs could not stratify for survival in non-immunotherapytreated patients $(\log -\operatorname{rank} \mathrm{P}=0.3039)$. Compared to the non-immunotherapy cohort, the immunotherapy cohort had higher kynurenine, glyceraldehyde 3 -phosphate, pyruvate and lactate, as well as succinate, fumarate, malate, oxaloacetate, aconitate and isocitrate (all $\mathrm{P}<0.05$ ). CONCLUSION: AAR of 3.5-7 could indicate an immunosuppressive microenvironment and needs further investigation to identify immunotherapy non-responders. The presence of elevated kynurenine, succinate, fumarate, malate and oxaloacetate suggests glutaminolysis related to immunotherapy. Research supported by the 2019 AACR-Bayer Innovation and Discovery Grant.

\section{IMMU-06. DISCERNING THE DIFFERENT BONE MARROW} IMMUNE LANDSCAPES

Michael Strong, Aqila Ahmed, Anda-Alexandra Calinescu, Xiaofeng Zhou, Tyler Robinson, Sabrina Rocco, Geoffrey Siegel, Gregory Clines, Bethany Moore, Evan Keller, and Nicholas Szerlip; University of Michigan, Ann Arbor, MI, USA

Roughly 400,000 people have bone metastases in the U.S. with the vast majority of these occurring in the spine. The etiology of bone metastasis still remains to be fully elucidated. This study explored the differences in immune landscape between long bone and spine that may contribute to higher rates of bone metastasis to the spine. Spines and femurs from male C57BL6/J mice $(\mathrm{N}=10)$ were processed for flow cytometry and immunophenotyping using Mass Cytometry by Time-Of-Flight (CyTOF). The cells were analyzed with CyTOF using a 33-surface protein marker mouse antibody panel. Spines $(\mathrm{N}=3)$ and femurs $(\mathrm{N}=2)$ from patients were analyzed with CyTOF using the Maxpar Complete Human T cell Immuno-Oncology Panel Set. There are global differences in the immune cell composition between the long bone and spine microenvironment. Flow cytometry revealed slight increases in the $\mathrm{CD} 45+$ and $\mathrm{Cd} 11 \mathrm{~b}+$ cell populations in the bone marrow of murine spines compared to murine long bone, which are markers for myeloid-derived suppressor cells (MDSCs). Using CyTOF, significant differences in the immune cell landscape between long bone and spine were observed. In the murine long bone, an increase in monocytes/macrophages, myeloid progenitors, granulocytic MDSCs, granulocytes, and mast cells was observed compared to the spine. In the murine spine an elevation of CD8a+ DC cells, classical monocytes, MDSCs, pDCs, memory T helper cells, and NK T cells was seen. Evaluation of human long bone and spine revealed similar trends with a predominance of myeloid progenitor cells and monocytes in the human vertebra compared to the human long bone marrow. Significant differences in the immune microenvironment exist between the spine and long bone 